\title{
Improvements in the Pigment-Based Inkjet Printing Performance of Poly(lactic acid) Nonwoven Fabric by Surface Coating Using Polyacrylamide
}

\author{
Ya-Dong $\mathrm{Li}^{\mathrm{a}}$, Zhanghua Zhao ${ }^{\mathrm{b}}$, Jinping Guan ${ }^{\mathrm{c}}$ and Ren-Cheng Tang ${ }^{\mathrm{d}}$ \\ College of Textile and Clothing Engineering, Soochow University, Suzhou 215123, China \\ a935309151@qq.com, b993859302@qq.com, cguanjinping@suda.edu.cn, dtangrencheng@suda.e \\ du.cn
}

\begin{abstract}
Polylactic acid (PLA) fiber is a biodegradable material, and can be used as an alternative to petrochemical-based fibers. Printed PLA nonwoven products have a wide market. The pigment inkjet printing of PLA nonwovens suffers from some defects such as poor outline sharpness of patterns, poor rub fastness, and degradation of PLA fiber in the process of heat curing. In this work, surface coating and modification using polyacrylamide (PAM) was employed to improve the pigment inkjet printing performance of PLA nonwovens. The effect of curing temperature on the direct ink jet printing performance was first discussed in terms of color depth, color fastness, penetration of pigment inks, and sharpness of printed pattern. Subsequently, the influence of the coating with different dosages of PAM on the physical properties, morphological structures, and printing features of the fabric was studied. The results showed that the coating with PAM obviously improved the apparent color depth and pattern sharpness of the printed fabric, and the curing at $135{ }^{\circ} \mathrm{C}$ after printing imparted good color fastness to the printed fabric.
\end{abstract}

Keywords: poly(lactic acid), nonwoven fabric, inkjet printing, pigment ink, coating, polyacrylamide.

\section{Introduction}

Polylactic acid (PLA) fiber has become a hot spot in textile industry because it can offer a renewable and biodegradable or recyclable alternative to petrochemical-based fibers, and widely used in non-woven fabrics [1]. PLA nonwovens possess unique characteristics such as low odor retention, hypoallergenic showing no evidence of skin sensitization, natural resistance to staining, outstanding water transport properties, good breathability and comfort, UV light resistance, and good thermo-regulating properties [2], and can be used to produce handbags, tablecloths, upholstery, feminine hygiene products, nappies, medical dressings and compresses, packaging, flower disk, etc. The printed PLA nonwoven products have a wide market. Pigment printing has an advantage of simple procedure without steaming and washing, and hence it is eco-friendly [3]. In recent years, digital inkjet printing has drawn great attraction due to its unique characteristics of short production cycle, low waste, abundant color gradation, high image quality, and better applicability for small batch and multi-variety production [4]. Pigment inkjet printing combines the two benefits of pigment printing and inkjet printing, and becomes a good selection for the printing of PLA nonwovens. The pigment inkjet printing of PLA nonwovens suffers from some defects such as poor rub fastness [5], degradation of PLA fiber at the stage of heat curing [5], and poor outline sharpness of patterns caused by the penetration of water-based ink droplets towards pattern edges due to the hydrophobic nature of PLA fiber, which is also found in the case of the inkjet printing of other polyester fabrics [6]. In this work, surface modification or coating using polyacrylamide (PAM) was employed to improve the pigment inkjet printing performance of PLA nonwoven fabric. 


\section{Materials and Methods}

\subsection{Materials}

PLA nonwoven fabric $\left(80 \mathrm{~g} / \mathrm{m}^{2}\right)$ was purchased from Shenzhen Shengdefu Investment Development Co. Ltd., China. Polyacrylamide (PAM; molecular weight, $1 \times 10^{7} \mathrm{~mol} / \mathrm{g}$ ) was bought from Sinopharm Chemical Reagent Shanghai Co. Ltd., Shanghai, China. Pigment inks were supplied by Shanghai YingGe Electronic Technologies Co. Ltd., China.

\subsection{Printing}

The printing process included the following steps: coating without and with PAM, drying, ink printing and curing. The PAM paste was prepared using different concentrations of PAM (0-4\% wt). The PLA fabric was impregnated in the PAM paste using a bath ratio of 1:50 (the ratio of fabric mass to PAM paste mass). After padded in two dips and nips using a two-roll laboratory padder, the sample was dried at $80{ }^{\circ} \mathrm{C}$ for $5 \mathrm{~min}$. Afterwards, the coated fabric was printed on the YTJ-500s digital inkjet printing machine, dried at $60{ }^{\circ} \mathrm{C}$ for $5 \mathrm{~min}$ and cured at a required temperature for $3 \mathrm{~min}$.

\subsection{Measurements}

The weight gain of the PLA fabric after coating with PAM was calculated according to the difference in the weight of the sample before and after coating as well as its original weight. The bending length and flexural rigidity of the PLA fabric coated PAM were evaluated according to GB/T 18318-2001. The surface morphology of the coated fabric was observed by a TM3030 tabletop scanning electron microscope. The visual picture of the printed fabric was recorded by a digital camera under natural light. The wash and rub fastness of the printed fabric was measured according to the standard test methods ISO 105-C06 and ISO 105-X12, respectively. The apparent color depth (K/S value) of PLA nonwoven fabric was evaluated by a HunterLab UltraScan PRO reflectance spectrophotometer. The penetration rate of pigment ink was calculated using the following equation:

$$
\text { Penetration }(\%)=100 \cdot(K / S)_{\mathrm{b}} /(K / S)_{\mathrm{f}}
$$

where $(K / S)_{\mathrm{b}}$ and $(K / S)_{\mathrm{f}}$ are the color depth of the back and front side of a printed fabric, respectively.

\section{Results and Discussion}

\subsection{Inkjet Printing of the PLA Nonwoven Fabric not Coated with PAM}

In order to discuss the feasibility of the direct inkjet printing for PLA nonwoven fabric not subjected to coating or sizing with PAM, the printed fabrics were cured at different temperatures. Fig. 1a shows that the color depth $(K / S)$ of the front side of the printed fabric increased with increasing curing temperature, which is probably due to the uniform distribution of pigments on the binder layer covering the PLA surface. As a result of this, the penetration percentage of pigment inks decreased. In the case of no coating with PAM, the sharpness of the printed pattern was poor or vague as shown in Fig. 1b. This indicates that the uncoated fabric displays the poor quality of pigment-based inkjet printing, and special measures (e.g., pre-coating or sizing) should be taken to solve this problem. Table 1 shows the color fastness of the printed fabrics. As the curing temperature increased, the wash fastness displayed no change, but the rub fastness was obviously improved because of the good film-forming ability of the binder contained in pigment ink at high temperatures [5]. Furthermore, as the curing temperature increased, the hand feeling of the fabric became stiffer due to the enhanced cross-linking ability of the binder [5], and the shrinkage of the fabric. Therefore, an appropriate curing should be performed at $135^{\circ} \mathrm{C}$.

\subsection{Inkjet Printing of the PLA Nonwoven Fabric Coating with PAM}

Fig. 2 shows the physical features of the fabric coated with PAM. As the dosage of PAM increased, the weight gain of the fabric increased linearly, and both of the bending and flexural rigidity also increased obviously. In particular, at 3\% and 4\% PAM, the bending and flexural rigidity became very high, implying that the resulting fabric has stiff hand feeling. Thus, the hand feeling of the fabric can be controlled by the dosage of PAM. It should be pointed out that the hand feeling of the printed fabric should be dependent on its end uses. 
Fig. 3 shows the SEM photos of the PLA nonwoven fabrics coated with different dosages of PAM. Compared with the uncoated fabric, the coated fabric displayed the pore structures into which PAM penetrated, and the morphological structure of the PLA fiber whose surface was covered by PAM. Moreover, with an increase in the dosage of PAM, the spaces between PLA fibers were more densely filled by PAM. The blocking of the pore structures by PAM would decrease the perpendicular penetration of pigment inks, and the diffusion of pigment inks towards the outside of the printed pattern on the fabric surface. Both of the two factors would improve the apparent color depth and sharpness of the printed fabric.
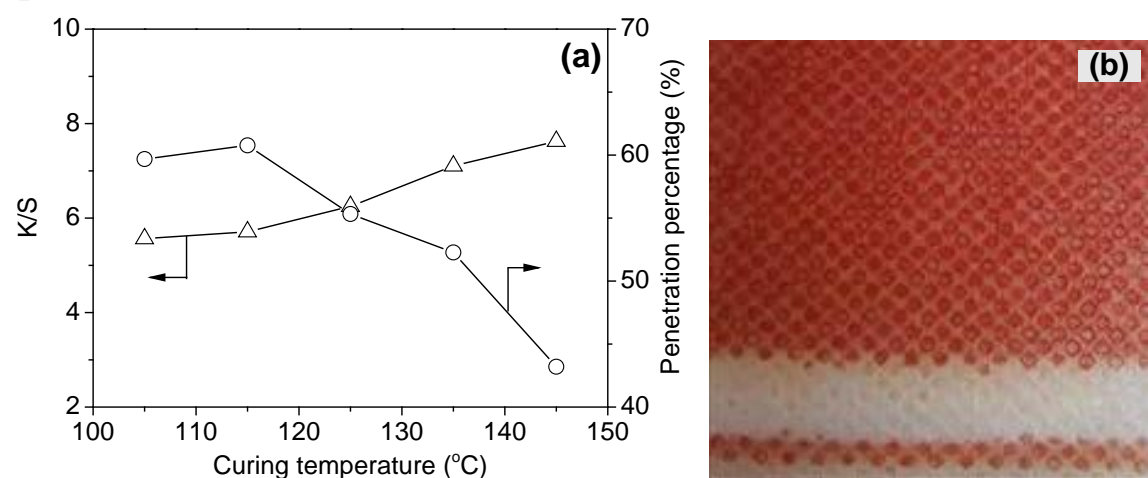

Fig. 1 Color characteristics of the printed PLA nonwoven fabrics without coating: color depth and penetration percentage (a), and the visual sample printed and cured at $135{ }^{\circ} \mathrm{C}$ (b)

Table 1 Effect of curing temperature on the color fastness of the printed PLA nonwoven fabrics

\begin{tabular}{|c|c|c|c|c|c|}
\hline \multirow{2}{*}{$\begin{array}{c}\text { Curing temperature } \\
\left({ }^{\circ} \mathrm{C}\right)\end{array}$} & \multicolumn{2}{|c|}{ Rub fastness } & \multicolumn{3}{c|}{ Wash fastness } \\
\cline { 2 - 6 } & Dry & Wet & Color change & \multicolumn{2}{|c|}{ Staining } \\
\cline { 3 - 6 } & & & & Polyester & Cotton \\
\hline 105 & 3 & $2-3$ & $4-5$ & 5 & $4-5$ \\
\hline 115 & $3-4$ & 3 & $4-5$ & 5 & $4-5$ \\
\hline 125 & $3-4$ & $3-4$ & $4-5$ & 5 & $4-5$ \\
\hline 135 & 4 & 4 & $4-5$ & 5 & $4-5$ \\
\hline 145 & 4 & 4 & $4-5$ & 5 & $4-5$ \\
\hline
\end{tabular}
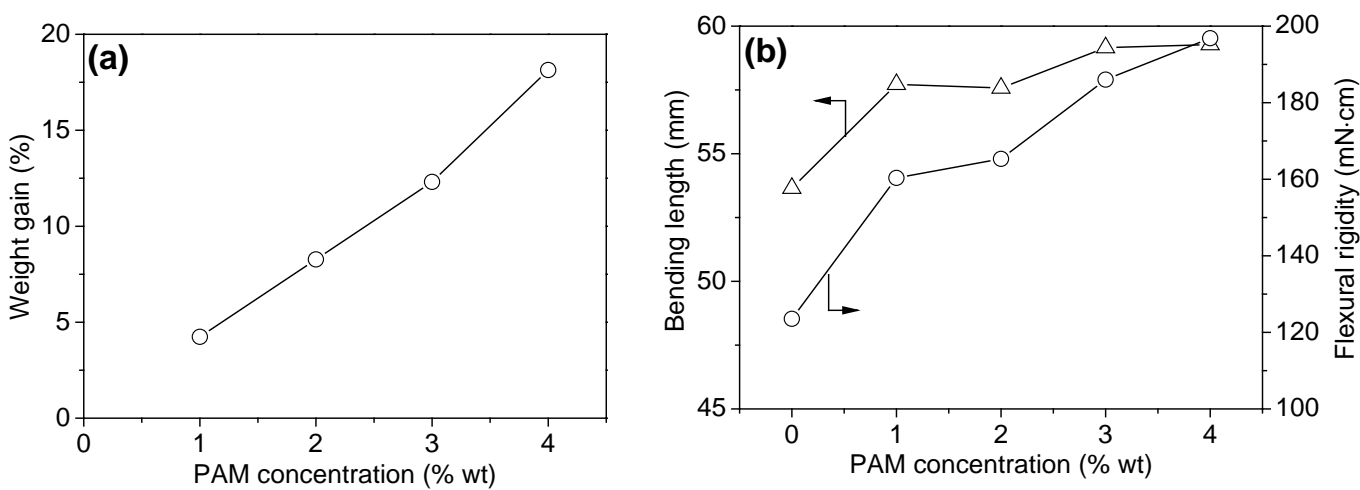

Fig. 2 Weight gain (a), and bending length and flexural rigidity (b) of the PLA nonwoven fabrics coated with different dosages of PAM
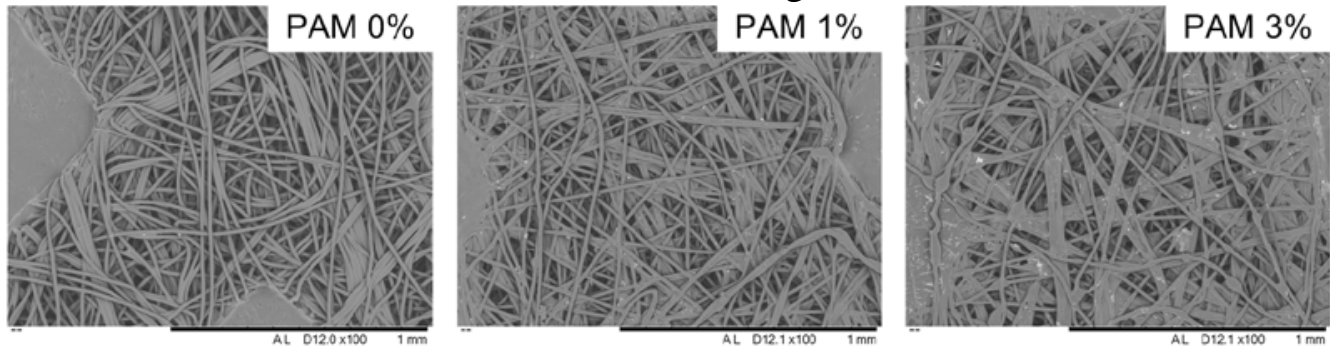

Fig. 3 SEM pictures of the PLA nonwoven fabrics coated with different dosages of PAM 
Fig. 4a shows that the color depth of the front side of the printed fabric increased with an increase in the dosage of PAM. This observation can be explained by the blocking of the pore structures of the fabric by PAM as shown in Fig. 3. From Fig. 4a, it is evident that the penetration percentage of pigment inks decreased obviously with increasing PAM dosage, as compared to that of the uncoated fabric. Fig. 4b shows that the printed pattern displayed a very clear sharpness. In comparison to the uncoated fabric (Fig. 1), the coated fabric exhibited higher color depth and sharper pattern outlines. The analyses of color fastness revealed that the printed fabric coated with 3\% PAM had the same fastness as the uncoated and printed fabric.
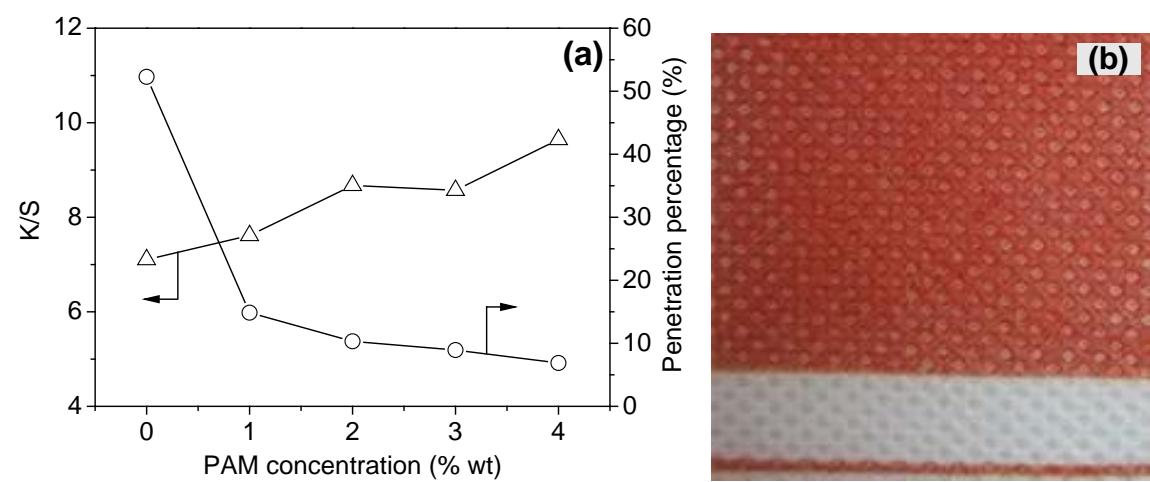

Fig. 4 Effect of PAM concentration on the color parameter of the coated and printed PLA nonwoven fabrics (a), and the visual sample coated, printed and cured at $135{ }^{\circ} \mathrm{C}$ (b)

\section{Conclusions}

In this work, surface coating using PAM was employed to improve the pigment inkjet printing performance of PLA nonwovens which was studied in terms of color depth, penetration of pigment inks, sharpness of printed pattern, and color fastness. Surface coating was found to increase the color depth and pattern sharpness of the printed fabric, decrease the penetration of pigment inks, and increase the bending length and flexural rigidity of the fabric with a negative impact on fabric softness. The curing at $135{ }^{\circ} \mathrm{C}$ after printing was able to provide good color fastness. The hand feeling of the fabric should be controlled by the dosage of PAM according to the end uses of PLA products.

\section{Acknowledgments}

This work was financially supported by the Joint Innovation Fund of Jiangsu Province of China (BY2014059-04).

\section{References}

[1] K.M. Nampoothiri, N.R. Nair, R.P. John, An overview of the recent developments in polylactide (PLA) research, Bioresource Technol. 101 (2010) 8493-8501.

[2] Information on http://www.natureworksllc.com/Product-and-Applications/Nonwovens.

[3] K. Fang, S. Wang, C. Wang, A. Tian, Inkjet printing effects of pigment inks on silk fabrics surface-modified with $\mathrm{O}_{2}$ plasma, J. Appl. Polym. Sci. 107 (2008) 2949-2955.

[4] A. Dehghani, F. Jahanshah, D. Borman, K. Dennis, J. Wang, Design and engineering challenges for digital inkjet printing on textiles, Int. J. Cloth. Sci. Tech. 16 (2008) 262-273.

[5] M.N. Karim, M. Rigout, S.G. Yeates, C. Carr, Surface chemical analysis of the effect of curing conditions on the properties of thermally-cured pigment printed poly(lactic acid) fabrics, Dyes Pigments 103 (2014) 168-174.

[6] L. Chen, C. Wang, A. Tian, M. Wu, An attempt of improving polyester inkjet printing performance by surface modification using $\beta$-cyclodextrin, Surf. Interface Anal. 44 (2012) 1324-1330. 